\title{
Discovery of an X-ray binary in the outer SMC wing ${ }^{\star}$
}

\author{
P. Kahabka and M. Hilker
}

\begin{abstract}
Sternwarte, Universität Bonn Auf dem Hügel 71, 53121 Bonn, Germany
e-mail: [pkahabka;mhilker]@astro.uni-bonn.de
\end{abstract}

Received 22 November 2004 / Accepted 21 December 2004

\begin{abstract}
The discovery of an X-ray binary RX J0209.6-7427 in the outer wing of the SMC is reported from two archival ROSAT PSPC observations. The data show variability in the X-ray light curve with a timescale of $\sim 40$ days that probably is related to the binary orbital period of the system. A $V=14 \mathrm{mag}$ star in the X-ray error circle of the source is the likely optical counterpart. The optical spectrum of the star shows $\mathrm{H} \alpha$ emission with an equivalent width of $-10.8 \pm 0.2 \AA$. The spectral type of the star is constrained as B0-B1.5IV-Ve and consistent with that of a Be star. RX J0209.6-7427 is therefore a Be-type $\mathrm{X}$-ray binary. The mean X-ray luminosity of the source is derived to be $\sim 1.0 \times 10^{38} \mathrm{erg} \mathrm{s}^{-1}$ (for a distance of $60 \mathrm{kpc}$ ), which is comparable to luminosities derived for Be-type X-ray binaries in the body of the SMC. This makes RX J0209.6-7427 the first candidate Be-type X-ray binary discovered in the outer SMC wing that directly extends into the Magellanic Bridge. This finding is consistent with the existence of a young ( 10-30 Myr) stellar population in the outer region of the SMC wing. Star formation due to an encounter of the SMC with the LMC and the Magellanic system with the Galaxy may explain this likely high-mass X-ray binary in an area far from the main body of the SMC.
\end{abstract}

Key words. Magellanic Clouds - X-rays: binaries - binaries: general - stars: individual: RX J0209.6-7427 stars: variables: general

\section{Introduction}

In recent years a considerable number $(\sim 45)$ of X-ray pulsars has been discovered in the field of the Small Magellanic Cloud (SMC), mostly in its body (e.g. Kahabka et al. 1999; Yokogawa et al. 2003; Haberl \& Pietsch 2004; Corbet et al. 2004; Popov $\&$ Raguzova 2004; Coe et al. 2005). For most of these X-ray pulsars an optical counterpart of the Be-type has been found in the X-ray error circle of the source. This points towards a high-mass X-ray binary nature for these sources. For a couple of the Be-type systems from $M A C H O$ and $O G L E$ observations, long-term light curves have been derived and periodicities in the range of days to tens of days have been found and interpreted as orbital periods (e.g. Coe \& Edge 2004).

The large number of observed Be-type high-mass X-ray binaries in the SMC allowed for an inference of a luminosity function and to compare it with the luminosity function of high-mass X-ray binaries derived for other galaxies (Grimm et al. 2003). The number of high-mass X-ray binaries scales with the recent star formation history of a sample of nearby late type/starburst galaxies (Grimm et al. 2003; see also Kahabka 2002 for the SMC). The recent star formation rate of the SMC, as inferred by Filipović et al. (1998) from the radio flux density of SMC supernova remnants, is $\sim 0.15 M_{\odot} \mathrm{yr}^{-1}$ and in

* Based on archival data from the ROSAT project. agreement with the star formation history derived by Harris \& Zaritsky (2004) (see also van der Heyden et al. 2004).

So far no efforts have been made to search for X-ray binaries located between the SMC and the LMC, in the so-called Magellanic Bridge. There is the SMC wing that extends to the east of the SMC (cf. Westerlund \& Glaspey 1971) and that contains a population of hot and young ( 10-60 Myr) stars (Demers \& Battinelli 1998; Courtès et al. 1995). This is also a favourable area to search for candidate high-mass X-ray binaries. The comparatively small number of stars in this area, together with the low probability of forming a neutron star Be star binary (e.g. van Bever \& Vanbeveren 1997), would not give a high probability of observing a Be-type X-ray binary in the Bridge. In case of a recent, enhanced formation of high-mass stars in the SMC wing, a few Be-type high-mass X-ray binaries may have formed and be observable.

A search for high-mass X-ray binaries in the SMC/LMC intercloud region (Bridge), therefore, may give valuable information about recent high-mass star formation in this region probably due to a close encounter of the SMC with the LMC and the Magellanic system with the Galaxy. In Sect. 2 the ROSAT observations of a candidate high-mass X-ray binary discovered in the outer SMC wing will be investigated. In Sect. 3 a likely optical counterpart of the source is discussed and its spectral confirmation presented in Sect. 4. Section 5 estimates the probability of observing a Be-type X-ray binary in the outer SMC 


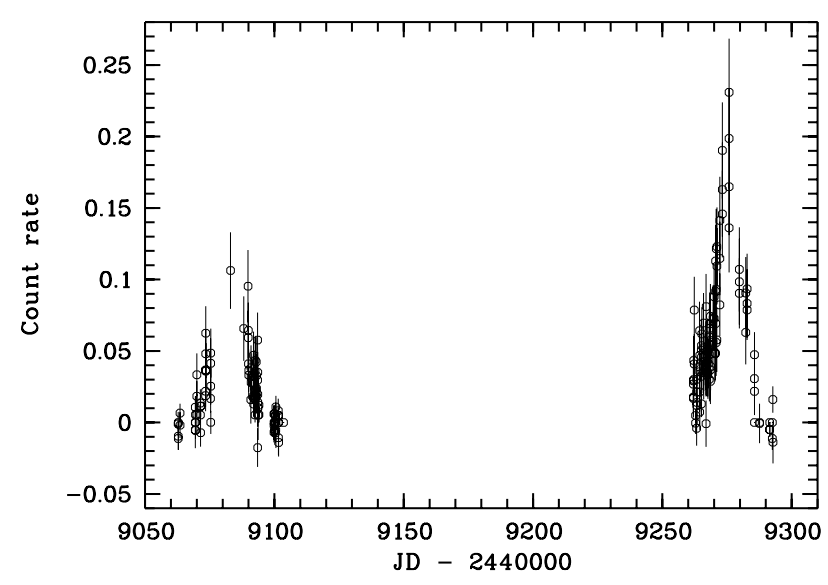

Fig. 1. Background subtracted, $\sim 0.9-2.5 \mathrm{keV}, \quad R O S A T$ PSPC lightcurve of RX J0209.6-7427 with a binsize of 5 min. Two X-ray bursts are seen, which are separated by $\sim 200$ days, the separation of the two ROSAT observations.

wing and in Sect. 6 implications for high-mass star formation in the SMC wing are discussed.

\section{The ROSAT observations of RX J0209.6-7427}

RX J0209.6-7427 has been discovered to be a bright, spectrally hard and variable X-ray source at a large off-axis angle of $37^{\prime}$ in two archival pointed ROSAT PSPC observations (ID wg900250p and wg900250p-1) of the Magellanic Bridge region (the first observation performed from March 16, 1993, 6:35 was until April 26, 1993, 1:15, and the second from Oct. 1, 1993, 1:31 until Nov. 1, 1993, 6:45). This observation of an H I cloud in the Bridge is discussed in Wang \& Ye (1996). The $\mathrm{X}$-ray source is contained in the ROSAT PSPC catalog (the 2RXP catalog) ${ }^{1}$ with the name 2RXP J020936.2-742707.

A sophisticated (local, map, and maximum likelihood) source detection algorithm (using the EXSAS software, Zimmermann et al. 1994) was applied to these data and a position derived for this source of RA $(\mathrm{J} 2000)=2^{\mathrm{h}} 9^{\mathrm{m}} 37.2^{\mathrm{s}}$, $\operatorname{Dec}(\mathrm{J} 2000)=-74^{\circ} 27^{\prime} 12^{\prime \prime}$. The $90 \%$ confidence positional error is $12^{\prime \prime}$, not taking into account a systematic positional error due to the point-spread function at a large off-axis angle of $37^{\prime}$ that could be as large as $\sim 30^{\prime \prime}$.

\subsection{X-ray outbursts}

Strong time variability was detected in the X-ray flux of this source. The binned light curve (with a bin size of $5 \mathrm{~min}$ and for the PSPC channel range 91-256) is given in Fig. 1.

Two X-ray bursts are seen, separated by $\sim 200$ days, the separation of the two ROSAT PSPC observations. Similar X-ray light curves have been observed from Be-type X-ray binaries in the LMC and the SMC. The outbursts in Be-type X-ray binaries are understood as due to the periastron passage of the neutron star and/or due to a mass eruption event of the Be star.

\footnotetext{
1 The second ROSAT source catalog of pointed observations, ROSAT consortium, ROSAT News 72, 25-May-2000.
}

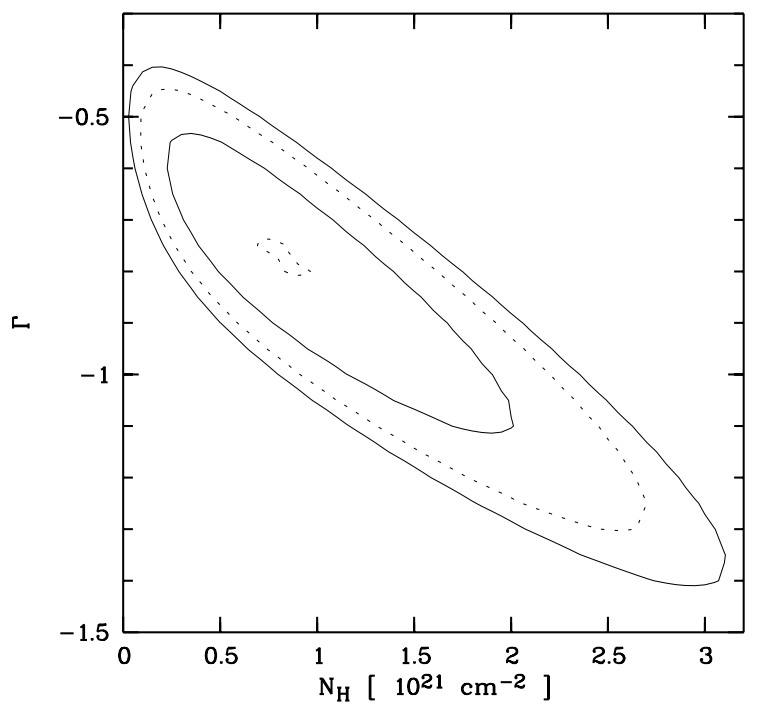

Fig. 2. 68, 95, and $99 \%$ chi-squared contours $N_{\mathrm{H}}$ of SMC wing gas versus powerlaw spectral slope $\Gamma$ for RX J0209.6-7427.

In order to search for a possible orbital period in this system, an FFT analysis was applied to the binned ROSAT PSPC data (Lomb 1976; Scargle 1982), and high power found at a period of $\sim 39$ days, which could be the orbital period of this system. But the time coverage of the two observations may be somewhat biased in favor of such a period and a conclusive answer for the existence of a $\sim 40$ day periodicity in the X-ray flux of this source still remains open.

\subsection{X-ray spectral slope, absorbing column density of SMC wing gas and $X$-ray luminosity}

The X-ray spectrum of the source is found to be hard. A powerlaw spectral model was applied to the merged data from both observations to constrain the powerlaw slope, the absorbing column density of the intervening gas and the unabsorbed flux and luminosity of the source. As the source is assumed to be located in the wing of the SMC, an absorber model consisting of a galactic and a SMC component was used. For the galactic component a column density of $3.8 \times 10^{20} \mathrm{~cm}^{-2}$ was assumed, as derived from a 21-cm Parkes map of the LMC/SMC field (Brüns et al. 2001). The column density of the SMC wing component was determined in the spectral fit assuming a low metallicity (reduced compared to the galactic metallicity by -0.5 dex, cf. Lee et al. 2005) consistent with other findings. From a 21-cm Parkes map of the SMC field a column of neutral hydrogen of $1.06 \times 10^{21} \mathrm{~cm}^{-2}$ was inferred in the direction of RX J0209.6-7427 (Brüns et al. 2001). A minimum chi-squared parameter search was applied to the data (cf. Fig. 2) and the SMC wing absorbing column density constrained to $0.8 \pm_{0.6}^{1.2} \times 10^{21} \mathrm{~cm}^{-1}$ at $68 \%$ confidence and to $0.8 \pm_{0.8}^{2.2} \times 10^{21} \mathrm{~cm}^{-1}$ at $99 \%$ confidence.

This is consistent with RX J0209.6-7427 being located outside the galactic gas layer and inside the SMC wing gas layer. The spectral slope is found to be rather flat $(-1.1<$ $\Gamma<-0.5$ at $68 \%$ confidence and $-1.4<\Gamma<-0.4$ at 
Table 1. Optical stars 1, 2, and 3 in the X-ray error circle of RX J0209.6-7427 and Star a in the field of view of the pre-image which shows in addition to Star $1 \mathrm{H} \alpha$ emission. Coordinates and magnitudes of the three optical stars ( 1 to 3 ) closest to the X-ray position (Coordinates from the USNO catalog), $B$ and $V$ magnitudes from this work, $R$ magnitudes from the GSC II catalog and $J, H$, and $K$ colors from the 2MASS catalog. Star 3 is not contained in the 2MASS cata$\log$ and $J, H$, and $K$ magnitudes are not given for this star accordingly.

\begin{tabular}{cccc}
\hline \hline Star & $\begin{array}{c}\text { RA } \\
(\mathrm{J} 2000)\end{array}$ & $\begin{array}{c}\text { Dec } \\
(\mathrm{J} 2000)\end{array}$ & $\begin{array}{c}\text { dist } \\
\left({ }^{\prime \prime}\right)\end{array}$ \\
\hline 1 & $2^{\mathrm{h}} 9^{\mathrm{m}} 34.51^{\mathrm{s}}$ & $-74^{\circ} 27^{\prime} 11.7^{\prime \prime}$ & 10.8 \\
2 & $2^{\mathrm{h}} 9^{\mathrm{m}} 40.79^{\mathrm{s}}$ & $-74^{\circ} 27^{\prime} 15.3^{\prime \prime}$ & 15.2 \\
3 & $2^{\mathrm{h}} 9^{\mathrm{m}} 49.46^{\mathrm{s}}$ & $-74^{\circ} 27^{\prime} 10.9^{\prime \prime}$ & 50 \\
$\mathrm{a}$ & $2^{\mathrm{h}} 8^{\mathrm{m}} 58.41^{\mathrm{s}}$ & $-74^{\circ} 24^{\prime} 44.5^{\prime \prime}$ & \\
\hline Star & $B$ & $V$ & $R$ \\
\hline 1 & $\lesssim 14.46$ & $\lesssim 14.45$ & $14.33 \pm 0.33$ \\
2 & $18.00 \pm 0.01$ & $17.33 \pm 0.01$ & $16.87 \pm 0.33$ \\
3 & $16.14 \pm 0.01$ & $16.26 \pm 0.01$ & $16.18 \pm 0.33$ \\
\hline Star & $J$ & $H$ & $K$ \\
\hline 1 & $14.29 \pm 0.04$ & $14.21 \pm 0.06$ & $14.05 \pm 0.07$ \\
2 & $16.03 \pm 0.08$ & $15.60 \pm 0.12$ & $15.28 \pm 0.18$ \\
\hline
\end{tabular}

99\% confidence) in agreement with spectral slopes derived for Be-type X-ray pulsars in the body of the SMC (Haberl \& Pietsch 2004). An unabsorbed flux of $2.3 \times 10^{-10} \mathrm{erg} \mathrm{cm}^{-2} \mathrm{~s}^{-1}$ is derived, which corresponds to an unabsorbed luminosity of $1.0 \times 10^{38} \mathrm{erg} \mathrm{s}^{-1}(0.1-2.4 \mathrm{keV})$ at a distance of $60 \mathrm{kpc}(\mathrm{cf}$. Demers \& Battinelli 1998, for distance determination of the intercloud association ICA 16 located in the SMC wing).

\section{Likely optical counterparts in the X-ray error circle}

RX J0209.6-7427 was observed at a comparatively large offaxis angle of $37^{\prime}$ in the PSPC. The positional uncertainty in the position is $12^{\prime \prime}$ as derived from a maximum likelihood analysis. There may be some additional systematic positional error due to the asymmetry of the point-spread function of the $P S P C$, but we assume that the positional error is not larger than $1^{\prime}$. We searched several catalogs (using VizieR Search at CDS), and with the GSC-II catalog ${ }^{2}$, found three stars within a search radius of 50". The star closest to the position of the X-ray source has a distance of 10.8" (Star 1, cf. Table 1). Two other close-by stars (Star 2 and Star 3, cf. Table 1) have distances of 15.2" and $50^{\prime \prime}$ respectively (see Fig. 3).

Using the 2MASS All-Sky Survey catalog (Cutri et al. 2003), we confirm the two closest stars for which $J, H$, and $K$ magnitudes are given. For the brightest star we derive $J-K$ as $0.24 \pm 0.08$ (cf. Table 1) which is in the range of $J-K$ colors found for Be-type stars (Coe 2000). The star is not in the

${ }^{2}$ The Guide Star Catalogue, Version 2.2.01, Space Telescope Science Institute (STScI) and Osservatorio Astronomico di Torino (2001), see also Acknowledgements.

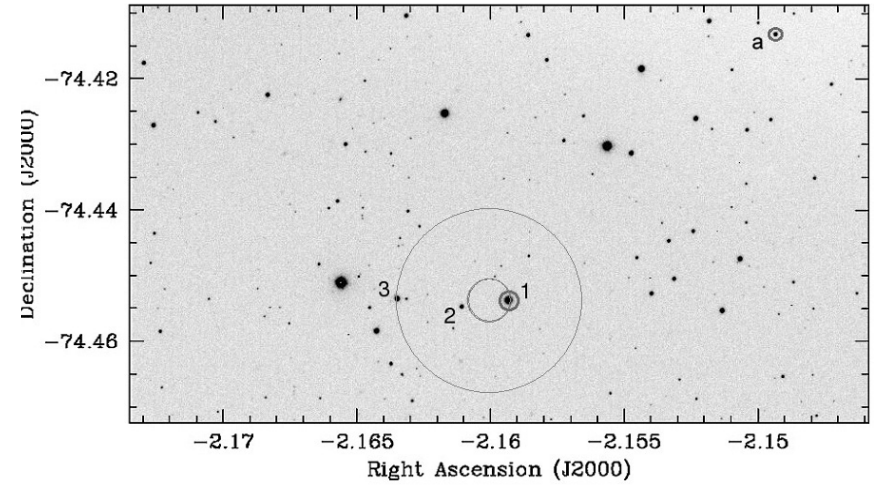

Fig. 3. Upper part of the pre-image which contains the X-ray source (circles of $12^{\prime \prime}$ and $50^{\prime \prime}$ radius). The bright $\sim 14$ mag star (Object 1 ) and two weaker stars (Objects 2 and 3 ) discussed in the text are labelled. In the upper north-west corner, the other $\mathrm{H} \alpha$ emitting star "a" is circled.

catalog of blue stars between the Magellanic Clouds by Demers \& Irwin (1991) for stars with a $V$ magnitude between 12.0 and 18.5 and a color index $B-V<0.0$. This is consistent with our data: $V \lesssim 14.5$ and $B-V \lesssim 0.01$ (cf. Table 1 ). Be stars have a color index $B-V \approx 0.0$ with $B-V>0.0$ in some cases (cf. Negueruela \& Coe 2002). Thus the star with $R=14.3$ may well be consistent with a Be star. An $R$ magnitude of 14.3 would also be consistent with $R$ magnitudes found for Be-type X-ray binaries in the Magellanic Clouds (cf. Negueruela \& Coe 2002), e.g. the LMC Be-type X-ray binary RX J0520.5-6932 has $R=14.3, B-V=0.02$, and it has an orbital period of 24.5 days. Thus it would resemble RX J0209.6-7427 assuming the 39-day periodicity seen in the X-ray flux would be the orbital period. For Star 2 in the X-ray error circle of RX J0520.5-6932, a $J-K$ color index of $0.75 \pm 0.20$ is derived. This value would be somewhat extreme for Be stars (cf. Coe 2000), while the $B-R$ color index of $1.1 \pm 0.3$ for this star would be outside the range of color indexes of the Be star high-mass X-ray binary sample of Negueruela \& Coe (2002). The most distant of these three stars, Star 3, has a color index $B-R=-0.04 \pm 0.33$. It is contained in the catalog of blue stars between the Magellanic Clouds of Demers \& Irwin (1991, with ID 589) and has a color index $B-V=-0.32$. We find $B-V=-0.12 \pm 0.01$. This star could be a Be star, e.g. similar to RX J0531.5-6518, Negueruela \& Coe (2002), but it has to be noted that the latter star is probably variable.

\section{Optical data and identification of the counterpart}

The optical stars 1 to 3 mentioned above are possible counterparts of the X-ray source, where position uncertainty is on the order of $\lesssim 50^{\prime \prime}$. The counterpart can be identified by its radial velocity, as well as by its spectral type and the strong $\mathrm{H} \alpha$ emission typical of Be stars. Other optical stars in the observed field can be used to set up a CMD and a radial velocity histogram. 

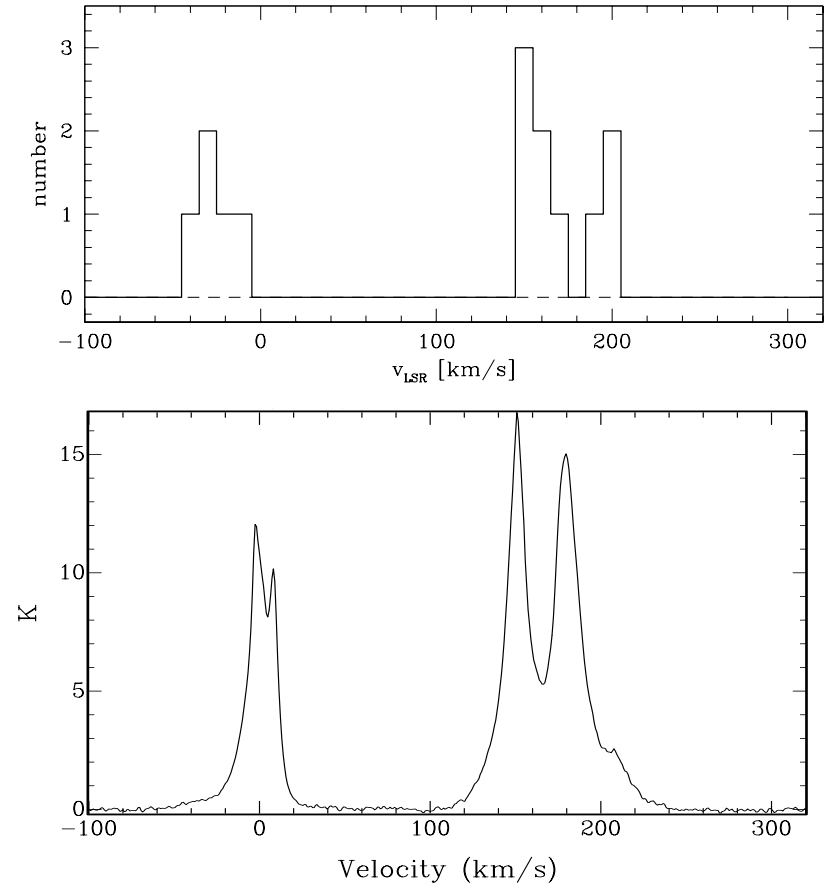

Fig. 4. Upper panel: velocity distribution of 14 stars for which their radial velocities with errors less than $50 \mathrm{~km} \mathrm{~s}^{-1}$ could be determined. Lower panel: velocities of the H I measured with Parkes at the location of RX J0209.6-7427.

\subsection{Observations and data reduction}

On July 26th 2004, spectra of candidate stars around the X-ray source have been taken with the VLT/UT4 at Paranal (ESO), Chile. The instrument in use was the FORS2 camera with the mask exchange unit MXU, and a $4 \times 4 \mathrm{k}$ MIT CCD attached. We chose the grism 600RI, which has a dispersion of $1.66 \AA$ pix $^{-1}$ and covers a wavelength range 5200-8400 $\AA$, thus containing the $\mathrm{H} \alpha$ line. Together with a seeing of $11^{\prime \prime} 8$ and a slit width of $1^{\prime \prime}$, the resulting resolution is $\sim 5.5 \AA$.

In the total field-of-view of FORS2, 20 objects were selected for the slit mask.

Figure 3 shows the upper part of the pre-image which contains the X-ray source (circle) and the integration time for the spectrum was 5 minutes, and the CCD frames were processed with standard IRAF routines. The signal-to-noise of the wavelength and flux calibrated, rebinned ( $2 \AA /$ pixel) spectra varied between 40 and 200 per pixel at $6800 \AA$ depending on the luminosity of the candidate stars.

\subsection{Radial velocities of target stars}

Radial velocities of the target objects were determined by cross-correlation (IRAF task FXCOR) with a radial velocity standard star observed with the same instrument setup.

Figure 4 shows the velocity distribution of 14 stars for which a radial velocity could be determined with errors less than $50 \mathrm{~km} \mathrm{~s}^{-1}$. All stars with velocities above $140 \mathrm{~km} \mathrm{~s}^{-1}$ are then SMC members, among them Star 1 and 3 from Fig. 3, with LSR (local standard of rest) velocities of $v_{\mathrm{LSR}}=149.6 \pm 12.5$ and $v_{\mathrm{LSR}}=172.3 \pm 24.4 \mathrm{~km} \mathrm{~s}^{-1}$, respectively. However, Star 2

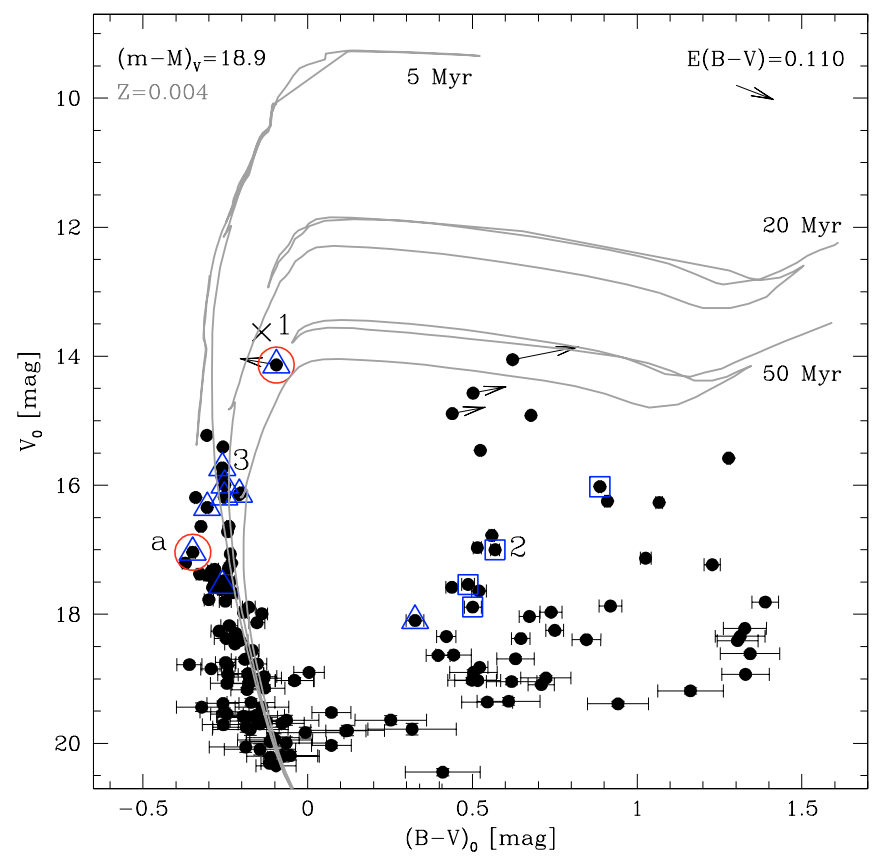

Fig. 5. Colour magnitude diagram of all point sources in the preimage. Confirmed members of the SMC are marked with triangles, foreground stars with squares. Candidate stars for the X-ray counterpart 1, 2, and 3 (see Fig. 3 and the further Be star "a") are labelled. The cross is the spectroscopically derived magnitude and color of Star 1. The isochrones were taken from Girardi et al. (2000). Their metallicity and ages are indicated in the plot.

is definitely a foreground star with $v_{\mathrm{LSR}}=-16.1 \pm 12.6 \mathrm{~km} \mathrm{~s}^{-1}$. Interestingly, one can see two velocity groups in the SMC around $v_{\mathrm{LSR}}=150$ and $190 \mathrm{~km} \mathrm{~s}^{-1}$. This corresponds well with the two velocity components of $\mathrm{HI}$ as measured with the Parkes beam at the location of RX J0209.6-7427 (cf. Fig. 4) and of gas found from HI surveys of the western Magellanic Bridge (Muller et al. 2003).

\subsection{CMD of the observed SMC wing field}

Short exposures in $B$ and $V$ were taken as pre-images for the spectroscopy under photometric conditions on July 23rd 2004. The magnitudes of all point sources were determined by aperture photometry and calibrated via Landolt standard stars, taken the same night. The brightest point sources were saturated either in $B$ or in $V$ depending on their colour. Among them, unfortunately, Star 1 was slightly saturated in $V$ (2 saturated pixels) and $B$ ( 4 saturated pixels).

In Fig. 5 the colour magnitude diagram of all point sources in the pre-image is shown. A reddening of $E_{B-V}=0.11$ was adopted, composed of a Galactic foreground reddening of $E_{B-V, \mathrm{MW}}=0.04 \mathrm{mag}$ (Schlegel et al. 1998) and an internal SMC reddening of $E_{B-V, S M C}=0.07$ mag. Clearly, the two Be stars belong to the blue main-sequence of young stars in the SMC wing (triangles mark other confirmed SMC members, squares foreground stars).

The main-sequence of the young stellar population in the SMC wing is well fit by isochrones of 5 to $20 \mathrm{Myr}$ 


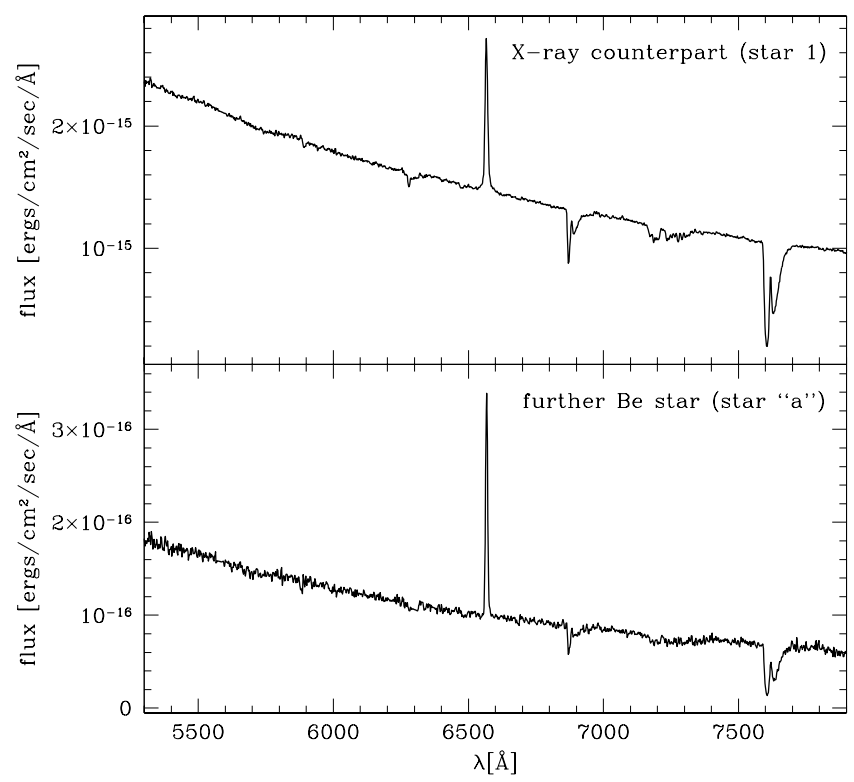

Fig. 6. Flux calibrated spectra of Star 1 (upper panel) and Star a surrounded by a circle in Fig. 3. Both stars show $\mathrm{H} \alpha$ emission. The strong absorptions red-wards $6800 \AA$ are from the night sky OH bands of the Earth's atmosphere.

(Girardi et al. 2000), assuming a metallicity of $Z=0.004$ for this region (SMC: $\sim-0.5$ dex, Lee et al. 2005), with reddening and distance as mentioned above. Older isochrones do not fit the upper main sequence stars at $V \sim 16$ mag.

\subsection{Star 1 as Be star is the likely counterpart}

Star 1 in the ROSAT error circle exhibits prominent $\mathrm{H} \alpha$ emission lines, which makes it the most probable X-ray counterpart. The flux calibrated spectrum is shown in the upper panel of Fig. 6. The equivalent width of the $\mathrm{H} \alpha$ line is $-10.8 \pm 0.2 \AA$, which is typical of Be stars (Coe et al. 2005). A value for the $\mathrm{H} \alpha$ equivalent width of $-10.8 \pm 0.2 \AA$, together with a likely orbital period of $\sim 40$ days, would be consistent with the dependence found for other SMC Be-type X-ray binaries (Coe 2000). RX J0209.6-7427 would also fit into the $\mathrm{H} \alpha / J-K$ color diagram populated by other SMC Be-type X-ray binaries (Coe 2000).

Assuming a distance modulus of $(m-M)_{V}=18.9 \mathrm{mag}$ (e.g. Harries et al. 2003), the absolute magnitude and colour of Star 1 is $M_{V} \simeq-4.76,(B-V) \leq-0.10$. From the position on the isochrone, the mass and spectral type can be estimated. The likely X-ray counterpart has a mass of 19 to $12 M_{\odot}$ for ages between 10 and $20 \mathrm{Myr}$. This age uncertainty comes from the unknown $(B-V)$ color due to saturation in both filters. In any case, its estimated location in the $\operatorname{CMD}(B-V \sim-0.2)$ is close to or just above the turnoff point of the isochrones depending on age. Thus it might still be a main-sequence star of luminosity class $\mathrm{V}$ (age $\lesssim 10 \mathrm{Myr}$ ) or rather a subgiant (class IV, $\sim 20 \mathrm{Myr}$ ). Its spectral type is around B0.

We also can use the optical spectrum of this star to set constraints on the spectral type. We make use of the Q method of Halbedel (1993), which requires that the $U, B$, and $V$ magnitude, corrected for extinction, has been determined. We extrapolate the spectral information towards the blue by adjusting reddened synthetic spectra from the stellar spectral library of Lejeune et al. (1997), while we can explain the observed spectra with the synthetic spectrum of a B star and the Planck approximation of a cool disk. We derive $Q=-0.85$, $M_{V} \approx-5.4$ (using a distance modulus of $18.9 \mathrm{mag}$ ). This gives a spectral type B0.5 to B1.5 within the range of spectral types found by Coe et al. (2005) for SMC Be-type X-ray binaries. Assuming the star is on the main-sequence, we infer a stellar mass of 13-10 $M_{\odot}$ making use of the spectral type mass dependence of Harmanec (1988). This is on the lower side for what was found using the isochrones.

\subsection{A further Be star in the optical field}

For another fainter star about 3.43 northwest of Star $1 \mathrm{H} \alpha$ line emission also was measured (cf. Fig. 3, small circle and Table 1 for the position of star given as Star a). This star is outside of the X-ray error circle and therefore not the optical counterpart of the X-ray source. Its flux calibrated spectrum is shown in the lower panel of Fig. 6 . It is a main-sequence star with $M_{V}=$ -1.85 and $(B-V)=-0.36$. From its absolute luminosity a mass between 8 and $9 M_{\odot}$ is estimated, and its spectral type is around B3. From the optical spectrum of the star we derive $Q=$ -0.70 and $M_{V}=-2.0$, in agreement with the findings from the CMD and somewhat underluminous for a main-sequence star (cf. Vacca et al. 1996).

\section{Estimated number of Be stars and Be-type X-ray binaries in the observed SMC wing field containing RX J0209.6-7427}

Recent observations of the SMC in X-rays have revealed 45 X-ray pulsars known in the SMC (Corbet et al. 2004; Haberl \& Pietsch 2004; Yokogawa et al. 2003). Haberl \& Pietsch (2004) give a Be star identification for $27 \mathrm{X}$-ray pulsars, which indicates that most of the SMC X-ray pulsars are high-mass X-ray binaries. They also give 65 candidates for high-mass X-ray binaries in the SMC. In the Galaxy there are 83 known highmass X-ray binaries (53 X-ray pulsars and 30 non pulsating sources). In addition there are 130 low-mass X-ray binaries in the Galaxy (Yokogawa et al. 2003; Liu et al. 2000, 2001). The positions of most of the high-mass X-ray binaries coincide with regions in the SMC where young (10-30 Myr) stars have been found (Yokogawa et al. 2003; Maragoudaki et al. 2001). Many of the observed Be-type X-ray binaries have low luminosities $\left(\sim 10^{35} \mathrm{erg} \mathrm{s}^{-1}\right)$ but pulsations are still detected in X-rays.

Several scenarii have been proposed to explain X-ray emission in Be-type systems, which are often characterized by outbursts with durations of tens of days and separated by up to hundreds of days. The two main scenarii are (1) periastron passage of a neutron star on an eccentric orbit through the disk of a Be star; and (2) eruptive mass-loss of a Be star. Some Be-type X-ray binaries show both types of outbursts (see also Stella et al. 1986 for a discussion of Class I and Class II outbursts). Be stars with type-1 stars showing outbursts and type- 2 stars showing high and low states are known 
(cf. Mennickent et al. 2002). There may also be a third type of variability in Be stars, called type-3, which is a periodic variability (Mennickent et al. 2002). The outburst periods are 16 to 332 days for type- 1 outbursts and 17 to 720 days for type- 3 outbursts. In case a Be star undergoes frequent eruptions, then Xray emission may be frequently observed, which may increase the number of Be-type X-ray binaries observed in a galaxy. It has been found that the number ratio $\mathrm{Be} /(\mathrm{Be}+\mathrm{B})$ increases with decreasing metallicity (Maeder et al. 1999), and this ratio was found to be $0.1,0.19,0.23$, and 0.39 for the interior, exterior of the Galaxy, the LMC, and the SMC respectively. One could speculate that this ratio $\mathrm{Be} /(\mathrm{Be}+\mathrm{B})$ is even larger in the Magellanic Bridge where the metallicity has been found to be very low ( -1.1 dex, Rolleston et al. 1999; see also Lehner 2002).

Mennickent et al. (2002) found a number of 1000 Be star candidates (out of 2 million stars) in the SMC using the $O G L E$ database. But there may be far more Be stars in the SMC. The number of Be-type X-ray binaries in the SMC can be estimated making use of an IMF $\xi$ (Scalo 1986)

$\xi(M)=\xi_{0} M^{-2.7}$.

The stellar mass of the SMC $M_{\text {stellar }}$ is assumed to be $2 \times 10^{9} M_{\odot}$ (Gardiner \& Noguchi 1996). It can be derived from the IMF

$M_{\text {stellar }}=\int_{0.1}^{50} \xi(M) M \mathrm{~d} M$.

Using

$\xi(M)=\xi_{0} M^{-2.7}$

one derives $M=\xi_{0} \int_{0.1}^{50} M^{-1.7} \mathrm{~d} M$, which gives $M=7.1 \xi_{0}$. With $M=M_{\text {stellar }}$, the stellar mass of the SMC, it follows that $\xi_{0}=2.8 \times 10^{8}$. The number of "B stars" in the spectral range O9 to $\mathrm{B} 2, N_{\mathrm{B}}$, is derived making use of the spectral type - mass relation of Harmanec (1988)

$N_{\mathrm{B}}=\xi_{0} \int_{10}^{17} M^{-2.7} \mathrm{~d} M$,

which gives $N_{\mathrm{B}}=2.0 \times 10^{6}$. Assuming that $40 \%$ of all B stars are Be stars and at most $1 \%$ of all these Be stars have a neutron star companion (van Bever \& Vanbeveren 1997), one gets at most 8000 Be-star neutron star binaries in the SMC. The fraction of these binaries that will appear as X-ray binaries depends on the orbital separation and the eccentricity but also on the lower luminosity threshold.

We note that associations in the SMC wing tip were found to be characterized by a shallower $\operatorname{IMF} \xi(M)=\xi_{0} M^{-(2.3 \pm 0.1)}$ (Grondin et al. 1992), which resembles a Salpeter type IMF $\xi(M)=\xi_{0} M^{-2.35}$.

With a number of $\sim 8 \times 10^{5}$ Be stars in the SMC and with assuming that at most $1 \%$ of the Be stars have a neutron star companion (van Bever \& Vanbeveren 1997), then there may exist at most a number of $\sim 8000 \mathrm{Be}$ star neutron star binaries in the SMC. In order to appear as an X-ray binary the orbit should not be too wide and the orbit should be eccentric. The number $\sim 8000$ would be very large compared to the number of $\sim 50$ Be-type X-ray binaries observed so far in the SMC. Scaling with the ratio of stellar mass of the SMC and the Magellanic Bridge of $\sim 10^{4}$ (assuming there are $\sim 10^{5}$ stars in the Bridge), one would expect at most one Be-type Xray binary in the Bridge.This number would not change much if the ratio $\mathrm{Be} /(\mathrm{Be}+\mathrm{B})$ is enhanced by a factor of 2 in the Bridge. As the number of stars in the Bridge is probably not well determined and as the stellar density in the SMC wing may differ from the mean stellar density in the Bridge, a more appropriate estimate of the number of Be-star neutron star binaries in the observed field of the SMC wing may be obtained using the stellar density maps of Gardiner \& Hatzidmitriou (1992). From their Fig. 5 it follows that at the location of RX J0209.6-7427 there are $~ 3000$ stars per square degree with $R<20$. Such stars correspond to a main-sequence mass of $\gtrsim 2.5 M_{\odot}$ at SMC distance. There are $3.5 \times 10^{7}$ stars in the SMC with mass larger than $2.5 M_{\odot}$. As there are $2 \times 10^{6}$ stars with mass in the range of Be stars, only 0.057 of these 3000 stars, i.e. 171 stars, are B stars. This number changes to $240 \mathrm{~B}$ stars if a shallower Salpeter IMF is chosen for the outer SMC wing (see Grondin et al. 1992). Further on numbers derived for a Salpeter IMF are also given in brackets. Assuming a Be-star fraction of 0.4 and a neutron star binary fraction of 0.01 , one obtains $0.68(0.96)$ Be-star neutron star binaries per square degree. As the PSPC field is $\sim 3$ square degrees there may be 2 (3) Be-star neutron star binaries in the field of view of the $P S P C$. If one of these were an X-ray binary then the observation of RX J0209.6-7427 could be explained. We note that due to the low stellar density, this number is much smaller than a mean number of $\sim 620 \mathrm{Be}$-star neutron star binaries per square degree as inferred assuming an area of $\sim 13$ square degrees for the SMC as follows from an H map and assuming column densities in excess of $10^{21} \mathrm{~cm}^{-1}$ (Brüns et al. 2001). The fact that a second Be star is found 3.4' distant from the likely optical counterpart can be compared with the expected number of 68 (96) Be stars per square degree and a mean distance of an expected closest Be star of 7.3'(6.1'). But consideration of just two Be stars does not allow us to derive constraints on the number density of Be stars in the observed field. We also note that our estimated number of Be stars and Be X-ray binaries is restricted to the spectral type range $\mathrm{O} 9$ to $\mathrm{B} 2$ and $\mathrm{Be}$ stars of a later spectral type have not been considered.

\section{High-mass star formation in the Magellanic Bridge}

The existence of a likely high-mass X-ray binary in the outer wing of the SMC that is part of the Magellanic Bridge indicates that there is recent $(\lesssim 30 \mathrm{Myr}$ ago) formation of high-mass stars in this region, and is also confirmed by young mainsequence, see CMD. This is supported by finding an expanding filamentary shell DEM 171 (Meaburn 1986; Graham et al. 2001) 200 pc distant from RX J0209.6-7427. Such a giant shell could be due to stellar winds and supernova explosions. The formation of high-mass stars in the Bridge region is also supported by finding young (10-60 Myr) stars (Demers \& Battinelli 1998) and the detection of molecular hydrogen in the direction of two early-type stars (Lehner 2002). Interestingly the neutral hydrogen column density due to SMC wing gas 
of $\sim 1.0 \times 10^{21} \mathrm{~cm}^{-2}$ at the location of RX J0209.6-7427 is near the critical threshold for star formation.

The Bridge is thought to be made up of material from the SMC pulled out by the LMC during a close encounter between both Clouds $\sim 0.2 \mathrm{Gyr}$ ago at a separation of $\sim 7 \mathrm{kpc}$ (see Gardiner \& Noguchi 1996; Putman 2004). Since that time, according to the simulations of Yoshizawa \& Noguchi (2003), the distance of the LMC and the SMC to the Galaxy was the least since $\sim 1.3 \mathrm{Gyr}$, which could account for recent star formation in the whole Magellanic system. The Bridge is also a site of current star formation, with the youngest stars having ages of 5 Myr (see discussion in Christodoulou et al. 1997). Grondin et al. (1992) found that the Initial Mass Function of the Bridge is shallower than that of the Galaxy or the Magellanic Clouds, which would favor the formation of massive stars and could indicate that cloud-cloud collisions are the dominant star formation process (cf. Scoville et al. 1986; Christodoulou et al. 1997; Putman 2004). The formation of massive stars may have been active only recently ( $\sim 16-25 \mathrm{Myr}$ ago), avoiding the enrichment of gas in heavy elements (Christodoulou et al. 1997).

\section{Conclusions}

For the first time a candidate X-ray binary RX J0209.6-7427 has been discovered in the outer SMC wing in archival ROSAT PSPC observations. The X-ray flux shows strong variability with a likely period of $\sim 40$ days, which could be the orbital period of the system. The X-ray spectrum can be fitted with a powerlaw with a photon index of $-\Gamma \sim(0.4-1.4)$ and an absorbing column density due to SMC wing gas of $N_{\mathrm{H}}=0.8 \pm_{0.8}^{2.2} \times 10^{21} \mathrm{~cm}^{-2}$, assuming a metallicity that is reduced compared to solar of -0.5 dex. The unabsorbed X-ray luminosity is $\sim 1.0 \times 10^{38} \mathrm{erg} \mathrm{s}^{-1}(0.1-2.4 \mathrm{keV})$.

A $~ 14$ mag star which shows $\mathrm{H} \alpha$ emission is in the X-ray error circle of RX J0209.6-7427, which is the likely optical counterpart of the source. From optical spectroscopy it is identified as a B0-B1.5IV-Ve star. Thus RX J0209.6-7427 is a Be-type X-ray binary in the outer SMC wing, and it is estimated that the number of Be-star neutron star binaries expected to exist in this region of the SMC and in the field of view of the ROSAT PSPC observation is consistent with the observation of RX J0209.6-7427.

Traces of recent star formation in the outer SMC wing may be due to an encounter between the SMC and the LMC and between the Magellanic system with the Galaxy. They are observed as expanding filamentary shells, young stars, hot gas and a high-mass X-ray binary.

Acknowledgements. The ROSAT project is supported by the MaxPlanck-Gesellschaft and the Bundesministerium für Forschung und Technologie (BMFT). The HI velocity profile as measured with Parkes at the location of RX J0209.6-7427 has been provided by C. Brüns. This research made use of the VizieR catalog access tool, CDS, Strasbourg, France. It also made use of the VizieR On-line Data Catalog: II/246 and of data products from the Two Micron All Sky Survey, which is a joint project of the University of Massachusetts and the Infrared Processing and Analysis Center/California Institute of Technology, funded by the National Aeronautics and Space Administration and the National Science Foundation. The Guide Star
Catalogue-II is a joint project of the Space Telescope Science Institute and the Osservatorio Astronomico di Torino. We thank the referee M. J. Coe for helpful comments.

\section{References}

Brüns, C., Kerp, J., Staveley-Smith, L. 2001, in Mapping the Hidden Universe: The Universe Behind the Milky Way - The Universe in HI, ed. R. C. Kraan Korteweg \& P. A. Henning ASP Conf., 218, 349

Chistodoulou, D. M., Tohline, J. E., \& Keenan, F. P. 1997, ApJ, 486, 810

Coe, M. J. 2000, in The Be Phenomenon in Early-Type Stars, IAU Coll. 175, ed. M. A. Smith \& H. Henrichs, ASP Conf. Proc., 214, 656

Coe, M. J., \& Edge, W. R. T. 2004, MNRAS, 350, 756

Coe, M. J., \& Edge, W. R. T., Galache, J. L., \& McBride, V. A. 2005, MNRAS, 356, 502

Corbet, R. H. D., Laycock, S., Coe, M. J., Marshall, F. E., \& Markwardt, C. B., 2004, in X-ray Timing 2003: Rossi and Beyond, ed. P. Kaaret, F. K. Lamb, \& J. H. Swank, AIP Conf. Proc., 714, 337

Courtès, G., Viton, M., Bowyer, S., et al. 1995, A\&A, 297, 338

Cutri, R. M., Skrutskie, M. F., van Dyk, S., et al. 2003, 2MASS AllSky Catalog of Point Sources

Demers, S., \& Irwin, M. J. 1991, A\&AS, 91, 171

Demers, S., \& Battinelli, P. 1998, AJ, 115, 154

Filipović, M. D., Pietsch, W., Haynes, R. F., et al. 1998, A\&AS, 127, 119

Gardiner, L. T., \& Hatzidimitriou, D. 1992, MNRAS, 257, 195

Gardiner, L. T., \& Noguchi, M. 1996, MNRAS, 278, 191

Garmany, C. D., Conti, P. S., \& Massey, P. 1980, ApJ, 242, 1063

Girardi, L., Bressan, A., Bertelli, G., \& Chiosi, C. 2000, A\&AS, 141, 371

Graham, M. F., Smith, R. J., Meaburn, J., \& Bryce, M. 2001, MNRAS, 326,539

Grimm, H.-J., Gilfanov, M., \& Sunyaev, R. 2003, MNRAS, 339, 793

Grondin, L., Demers, S., \& Kunkel, W. E. 1992, AJ, 103, 1234

Haberl, F., \& Pietsch, W. 2004, A\&A, 414, 667

Halbedel, E. M. 1993, PASP, 105, 465

Harmanec, P. 1988, Bull. Astron. Inst. Czechosl., 39, 329

Harries, T. J., Hilditch, R.W, \& Howarth, I. D. 2003, MNRAS, 339, 157

Harries, J., \& Zaritzky, D. 2004, AJ, 127, 1531

Kahabka, P., Pietsch, W., Filipović, M. D., \& Haberl, F. 1999, A\&AS, 136,81

Kahabka, P. 2002, A\&A, 388, 100

Lee, J.-K., Rolleston, W. R. J., Dufton, P. L., \& Ryans, R. S. I. 2005, A\&A, 429, 1025

Lehner, N. 2002, ApJ, 578, 126

Lejeune, T., Cuisinier, F., \& Buser, R. 1997, A\&AS, 125, 229

Liu, Q. Z., van Paradijs, J., \& van den Heuvel, E. P. J. 2000, A\&AS, 147,25

Liu, Q. Z., van Paradijs, J., \& van den Heuvel, E. P. J. 2001, A\&A, 368,1021

Lomb, N. R. 1976, Ap\&SS, 39, 447

Maeder, A., Grebel, E. K., \& Mermilliod, J.-C. 1999, A\&A, 346, 459

Maragoudaki, F., Kontizias, M., Morgan, D. H., et al. 2001, A\&A, 379,864

Meaburn, J. 1986, MNRAS, 223, 317

Mennickent, R. E., Pietrzyński, G., Gieren W., \& Szewczyk, O. 2002, A\&A, 393, 887 
Muller, E., Staveley-Smith, L., \& Stanimirović, S. 2003, MNRAS, 339,105

Negueruela, I., \& Coe, M. J. 2002, A\&A, 385, 517

Popov, S. B., \& Raguzova, N. V. 2004 [arXiv: astro-ph/0405633]

Putman, M. E. 2004, in High-Velocity Clouds, ed. H. van Woerden, P. B. Wakker, U. J. Schwarz, \& K. S. de Boer, Astrophysics and Space Science Library (Dordrecht: Kluwer Academic Publishers), 312,101

Rolleston, W. R. J., Dufton, P. L., McErlean, N. D., \& Venn, K. A., 1999, A\&A, 348, 728

Scalo, J. M. 1986, Fund. Cosmic Phys., 2, 1

Scargle, J. D. 1982, ApJ, 263, 835

Schlegel, D. J., Finkbeiner, D. P., \& Davis, M. 1998, ApJ, 500, 525
Scoville, N. Z., Sanders, D. B., \& Clemens, D. P. 1986, ApJ, 310, L77

Stella, L., White, N. E., \& Rosner, R. 1986, ApJ, 308, 669

Vacca, W. D., Garmany, C. D., \& Shull, J. M. 1996, ApJ, 460, 914

van Bever, J., \& Vanbeveren, D. 1997, A\&A, 322, 116

van der Heyden, K. J., Bleeker, A. M., \& Kaastra, J. S. 2004, A\&A, 421, 1031

Wang, Q. D., \& Ye, T. 1996, New. Astr., 1, 245

Westerlund, B. E., \& Glaspey, J. 1971, A\&A, 10, 1

Yokogawa, J., Imanishi, K., Tsujimoto, M., \& Koyama, K. 2003, PASJ, 55, 161

Yoshizawa, A. M., \& Noguchi, M. 2003, MNRAS, 339, 1135

Zimmermann H. U., Becker W., Belloni T., et al. 1994, MPE Rep., 257 